\title{
Importance of AT1 and AT2 receptors in the nucleus of the solitary tract in cardiovascular responses induced by a high-fat diet
}

\author{
Guilherme F. Speretta ${ }^{1,2} \cdot$ Prashant J. Ruchaya $^{1} \cdot$ Maria A. Delbin ${ }^{3} \cdot$ Mariana R. Melo $^{1} \cdot$ Hongwei $^{4}{ }^{4} \cdot$ \\ José V. Menani ${ }^{1}$ - Colin Sumners ${ }^{5} \cdot$ Eduardo Colombari $^{1} \cdot$ Mirian Bassi $^{1}$ • Débora S. A. Colombari ${ }^{1}$
}

Received: 30 January 2018 / Revised: 27 June 2018 / Accepted: 26 July 2018 / Published online: 10 January 2019

(c) The Japanese Society of Hypertension 2019

\begin{abstract}
A high-fat diet (HFD) induces an increase in arterial pressure and a decrease in baroreflex function, which may be associated with increased expression of angiotensin type 1 receptor (AT1R) and pro-inflammatory cytokine genes and reduced expression of the angiotensin type 2 receptor (AT2R) gene within the nucleus of the solitary tract (NTS), a key area of the brainstem involved in cardiovascular control. Thus, in the present study, we evaluated the changes in arterial pressure and gene expression of components of the renin-angiotensin system (RAS) and neuroinflammatory markers in the NTS of rats fed a HFD and treated with either an AT1R blocker or with virus-mediated AT2R overexpression in the NTS. Male Holtzman rats (300-320 g) were fed either a standard rat chow diet (SD) or HFD for 6 weeks before commencing the tests. AT1R blockade in the NTS of HFD-fed rats attenuated the increase in arterial pressure and the impairment of reflex bradycardia, whereas AT2R overexpression in the NTS only improved the baroreflex function. The HFD also increased the hypertensive and decreased the protective axis of the RAS and was associated with neuroinflammation within the NTS. The expression of angiotensin-converting enzyme and neuroinflammatory components, but not AT1R, in the NTS was reduced by AT2R overexpression in this site. Based on these data, AT1R and AT2R in the NTS are differentially involved in the cardiovascular changes induced by a HFD. Chronic inflammation and changes in the RAS in the NTS may also account for the cardiovascular responses observed in HFD-fed rats.
\end{abstract}

Keywords Baroreflex $\cdot$ Brainstem $\cdot$ Cytokines $\cdot$ Obesity $\cdot$ Renin-angiotensin system

Supplementary information The online version of this article (https:// doi.org/10.1038/s41440-018-0196-0) contains supplementary material, which is available to authorized users.

Débora S. A. Colombari

deborac@foar.unesp.br

1 Department of Physiology and Pathology, School of Dentistry, UNESP—São Paulo State University, Araraquara, SP, Brazil

2 Department of Physiological Sciences,Biological Sciences Centre, Federal University of Santa Catarina (UFSC), Rua Roberto Sampaio Gonzaga s/n, 88040-900, Trindade, Florianópolis, SC, Brazil

3 Department of Structural and Functional Biology, Institute of Biology, UNICAMP-University of Campinas, Campinas, SP, Brazil

4 School of Biotechnology, Southern Medical University, Guangzhou, China

5 Department of Physiologyand Functional Genomics and McKnight Brain Institute, College of Medicine, University of Florida, Gainesville, FL, USA

\section{Introduction}

An understanding of the comorbidity of obesity and hypertension has been the focus of several studies during the last few years, as previously reviewed [1,2]. Different mechanisms have been described as facilitating obesityinduced hypertension, including increased activity of the central and peripheral renin-angiotensin systems (RAS), chronic inflammation, and high levels of leptin [3-6]. Central effects of these factors elicit increased sympathetic nerve activity (SNA) and arterial pressure in obesity $[1,5,7]$. In addition, obesity alone or in combination with hypertension decreases baroreflex sensitivity, impairing the control of SNA [5, 8-10].

However, the roles of specific brain cardiovascular control centers and the contributions of factors, such as the RAS and pro-inflammatory cytokines (PIC) at those central nervous system (CNS) sites to obesity-induced hypertension have not been established. One of the most important brain areas involved in cardiovascular regulation is the 
nucleus of the solitary tract (NTS) [11-13], which is located in the dorsal hindbrain. The NTS is the first synaptic site of baroreceptor afferents in the CNS [14] and plays major roles in the impaired baroreflex function and sympathoexcitation associated with hypertension, including obesity-induced hypertension [10, 12, 15]. Angiotensin type 1 receptor (AT1R) is expressed at high levels in the NTS $[12,16]$ and is a significant contributor to these deleterious effects, as its activation by angiotensin II (ANG II) decreases baroreflex sensitivity [17]. Furthermore, the increase in arterial pressure and reduction in baroreflex sensitivity induced by a high-fat diet (HFD) are associated with increased levels of the AT1R mRNA in the NTS [5], suggesting that central AT1R might be involved in these responses. Considering that the centrally mediated hypertensive effects of ANG II/ AT1R involve the induction of neuroinflammation [1], unsurprisingly, an intracerebroventricular (icv) infusion of pentoxifylline, an inhibitor of synthesis of the PIC tumor necrosis factor alpha (TNF)- $\alpha$, or minocycline, an antibiotic with anti-inflammatory effects, attenuates the HFD-induced sensitization of ANG II-elicited hypertension [18].

The cardiovascular effects of ANG II on the NTS are not restricted to its signaling through AT1R. A significant population of angiotensin type 2 receptors (AT2R) is present within the cardioregulatory areas of the NTS [19, 20], and in contrast to AT1R, increased activity of AT2R in this area (following its overexpression) has been shown to restore baroreflex function [12, 13] and lower blood pressure [12] in animal models of neurogenic hypertension. However, the role of these NTS AT2R in obesity-induced hypertension is not known.

Taking into account current knowledge, the major aim of the present study was to evaluate the contributions of NTS AT1R and AT2R to mediating the increase in arterial pressure and the impairment of baroreflex function induced by a HFD. Furthermore, gene expression of RAS components and PICs in the NTS was also investigated in rats fed a HFD.

\section{Methods}

\section{Animals}

Male Holtzman rats weighing 300-320 g were used in this study. The animals were maintained in individual polypropylene cages in a room with a controlled temperature $\left(23 \pm 2{ }^{\circ} \mathrm{C}\right)$ and humidity $(55 \pm 10 \%)$ with food (please see the composition below) and water provided ad libitum. Lights were on from 7:00 a.m. to 7:00 p.m. The Ethics Committee for Animal Care and Use of the Dental School of Araraquara, UNESP, approved the experimental protocols used in the present study (\# 20/2013).

\section{Diet}

The experimental groups received either the standard rat chow diet (Biobase, Águas Frias, SC, Brazil), named the standard diet (SD), or HFD for 6-7 weeks. A bromatological analysis (Engeali, São José do Rio Preto, SP, Brazil) determined that the SD contained $22 \mathrm{~g}$ of protein, $48 \mathrm{~g}$ of carbohydrates, $4 \mathrm{~g}$ of total fat, and $8 \mathrm{~g}$ of fiber per $100 \mathrm{~g}$ of diet. The HFD comprised standard rat chow plus peanuts, milk chocolate, and sweet biscuits in a proportion of 3:2:2:1 and contained $13 \mathrm{~g}$ of protein, $40 \mathrm{~g}$ of carbohydrate, $19 \mathrm{~g}$ of total fat, and $4 \mathrm{~g}$ of fiber per $100 \mathrm{~g}$ of diet, as described previously [5]. The caloric values of the diets were $\sim 2.25 \mathrm{kcal} / \mathrm{g}$ for the SD and $3.82 \mathrm{kcal} / \mathrm{g}$ for the HFD [5].

\section{Anesthesia and euthanasia}

Rats were anesthetized with a mixture of ketamine [80 mg/ $\mathrm{kg}$ of body weight (b.wt.), intraperitonially (i.p.); Cristalia, Itapira, SP, Brazil] and xylazine $(7 \mathrm{mg} / \mathrm{kg}$ of b.wt., i.p.; Agener Union, Embu, SP, Brazil) prior to surgery. Following the surgeries, animals received a prophylactic dose of penicillin (50,000 IU, intramuscularly) and a dose of the anti-inflammatory agent ketoprofen $(1 \mathrm{mg} / \mathrm{kg}$ of b.wt., subcutaneously). At the end of the experiments, depending on the protocol (see below), rats were euthanized by placing them under deep anesthesia with sodium thiopental (70 mg/kg of b.wt., i.p.) and transcardially perfused with cold $0.9 \%$ saline followed by a $4 \%$ paraformaldehyde (PFA; Sigma, St. Louis, MO) solution in 0.1 M PB (pH 7.4) for immunohistochemistry or with isoflurane $\left(5 \%\right.$ in $\left.100 \% \mathrm{O}_{2}\right)$ and immediately decapitated for RT-PCR studies.

\section{Acute arterial pressure recordings, baroreflex tests, and cardiovascular variability}

Rats were anesthetized as described above, and a catheter was inserted into the abdominal aorta through the femoral artery for recordings of the pulsatile arterial pressure (PAP), mean arterial pressure (MAP) and heart rate (HR); a second polyethylene tube was inserted into the femoral vein for drug administration. The arterial and venous catheters were tunneled subcutaneously and exposed on the back of the rat. The arterial catheter was connected to a Statham Gould (P23 Db; El Segundo, CA, USA) pressure transducer coupled to a preamplifier (model ETH-200 Bridge Bio Amplifier, Chicago, IL, USA) that was connected to a Powerlab computer data acquisition system (model Powerlab 16SP, ADInstruments, Colorado Springs CO, USA) to record PAP, MAP, and HR in conscious unrestrained, freely moving animals, as described previously [6, 12, 20]. Twenty-four hours after catheter implantation, after $30 \mathrm{~min}$ of recording under baseline conditions, the bradycardic and 
tachycardic reflex responses of conscious rats were assessed in the same animals following an i.v. injection of phenylephrine $(5 \mu \mathrm{g} / \mathrm{kg}$, b.wt.) or sodium nitroprusside (SNP; $30 \mu \mathrm{g} / \mathrm{kg}$, b.wt.), respectively, with a $10 \mathrm{~min}$ interval, and the baroreflex sensitivity was analyzed using previously described methods $[5,12,21]$.

Systolic blood pressure (SBP) variability analysis was performed using customized software (CardioSeries v2.4-http://www.danielpenteado.com), as described previously $[5,12]$. Briefly, the spectra were integrated into low-frequency (LF; $0.2-0.75 \mathrm{~Hz}$ ) and high-frequency $(0.75-3 \mathrm{~Hz})$ bands, and results are presented as absolute values $\left(\mathrm{mmHg}^{2}\right)$.

\section{Telemetry recording of arterial pressure}

Rats were anesthetized as described above, and telemetry transmitters (TA11PAC40, Data Sciences International, St. Paul, MN) were implanted into the abdominal aorta as previously described [21]. MAP and HR were recorded continuously (sampling rate of $1 \mathrm{kHz} ; 1$ min every $5 \mathrm{~min}$ ) for a 24-h period, weekly for 6 weeks.

\section{NTS cannula and drug injection}

Anesthetized rats were placed in a stereotaxic apparatus (David Kopf Instruments, Tujunga, CA) and stainless steel cannulas were chronically implanted bilaterally in the intermediate NTS (iNTS) as described previously [22]. Rats were allowed to recover for 7 days before drug injection into the NTS. Losartan (AT1R antagonist; Dupont; Wilmington, DE, USA) was dissolved in saline. Bilateral injections of losartan $(10 \mu \mathrm{g} / 100 \mathrm{nl})$ were performed using $5 \mu \mathrm{l}$ Hamilton syringes connected by polyethylene tubing (PE 10) to the injector needles $(1.2 \mathrm{~mm}$ longer than the guide cannula). The dose of losartan chosen was based on a previous study by Vieira et al. [23].

At the end of the experiments, 2\% Evans blue solution $(100 \mathrm{nl})$ was injected bilaterally into the NTS. Thereafter, the animals were deeply anesthetized as described above and transcardially perfused. The brains were frozen, cut into $50-\mu \mathrm{m}$ coronal sections using a cryostat (Leica, CM1850, Wetzlar, Hesse, Germany), stained with Giemsa stain and analyzed under a light microscope (Leica DM5500 B, Wetzlar, Hesse, Germany) to trace the location of bilateral injections into the iNTS.

\section{In vivo gene transfer in the NTS}

Rats were anesthetized as described above and placed in a stereotaxic frame (David Kopf Instruments, Tujunga, CA). A partial craniotomy of the occipital bone was performed, and the dorsal surface of the brainstem was exposed.
AAV2-CBA-GFP or AAV2-CBA-AT2R was microinjected at five different sites along the NTS as described previously $[12,21]$. Each microinjection consisted of $150 \mathrm{nl}$ vector/site (GFP, $1.8 \times 10^{12}$ genome copies $(\mathrm{gc}) / \mathrm{ml}$ and AT2R, $1.2 \times$ $\left.10^{12} \mathrm{gc} / \mathrm{ml}\right)$. AAV2-CBA-GFP and AAV2-CBA-AT2R were constructed as described previously, and these vectors elicit gene transduction primarily in neurons [24].

\section{Blood and tissue collection}

The animals were anesthetized as described above and immediately decapitated. Blood was collected in EDTAcoated tubes for plasma leptin analyses and non-coated tubes for lipid profile measurements as described previously [5]. After blood collection, the brains were quickly removed, immediately frozen on dry ice and stored at $-80^{\circ} \mathrm{C}$ for subsequent mRNA extraction (described below). Finally, retroperitoneal (RET), epididymal (EPI), and mesenteric (MES) white adipose tissue samples were dissected and weighed.

\section{qRT-PCR analyses of the NTS}

The NTS was removed with a micropunch and the assistance of a surgical microscope using the area postrema (AP) and calamus scriptorius as reference sites. qRT-PCR analyses in the NTS were performed as described in detail in a previous study [21]. We evaluated the levels of the tumor necrosis factor $\alpha$ (TNF- $\alpha$; Rn99999017_m1), interleukin-6 (IL-6; Rn01410330_m1), angiotensin type-1 receptor (AT1R; Rn01435427_m1), angiotensin type-2 receptor (AT2R; Rn00560677_s1), Mas receptor (Mas; Rn00562673_s1), angiotensin-converting enzyme (ACE; Rn00561094_m1), and angiotensin-converting enzyme 2 (ACE2; Rn01416293_m1) mRNAs in the NTS. Expression patterns of the genes of interest were normalized to constitutively expressed GAPDH (Rn99999916_s1), and relative expression was quantified using the $2^{-\Delta \Delta \mathrm{Ct}}$ method [25].

\section{Immunohistochemistry}

Rats were deeply anesthetized with and transcardially perfused as described above. Brainstems were removed, frozen and four sets of coronal sections $(30 \mu \mathrm{m})$ of the brainstem were sectioned on a cryostat (Leica, CM1850, Wetzlar, Hesse, Germany). Immunohistochemistry was performed on every fourth section (each $120 \mu \mathrm{m}$ ) as previously described [26]. A rabbit anti-Iba-1 primary antibody (1:300; Wako Chemicals, Richmond, VA, USA) and mouse antiGFAP antibody (1:400, Calbiochem, Billerica, MA, USA) were used to label microglia and astrocytes, respectively, in the same sections. Iba-1 and GFAP immunofluorescence 
were detected using a fluorescence microscope (Leica DM5500 B Leica, Wetzlar, Hessen, Germany) with the appropriate filters.

The number of Iba-1-positive cells or the area of GFAP staining in the NTS, and in the hypoglossal nucleus (XII) and the AP, which served as control areas, were assessed using Leica Application Suite Advanced Fluorescence (Leica DM5500 B, Wetzlar, Hessen, Germany). Images were captured at $\times 20$ magnification at $120 \mu \mathrm{m}$ intervals as defined above. The iNTS was located throughout the AP level, above the dorsal motor nucleus of the vagus, whereas the commissural NTS (cNTS) was located from the obex to $500 \mu \mathrm{m}$ caudal to the obex, above the dorsal motor nucleus of the vagus. For the assessment of microglial number, images of Iba-1-stained sections were captured in $20 \mu \mathrm{m} \mathrm{z}$ stacks (containing 20 images) and Iba-1-positive cells were manually counted in matched representative sections of the NTS, XII and AP. For the assessment of the area of GFAP staining, images were converted into grayscale and binary formats and thresholds for black and white balance were adjusted to the same level in matched representative sections of the NTS, XII, and AP; the area of GFAP staining was presented as a gray value/section. For the iNTS and the XII, counts and gray values were recorded from both sides and averaged.

\section{Insulin tolerance test}

Insulin sensitivity was measured by performing an insulin tolerance test (ITT), and the rate constant for plasma glucose disappearance (Kitt) was calculated using a previously described method [5, 27]. Recombinant human insulin (Novolin R, Novo Nordisk, Montes Claros, MG, Brazil) was administered at a dose of $2.0 \mathrm{U} / \mathrm{kg}$, i.p. Blood samples were collected from the tail vein before (time 0 ) and 5, 10, 15, 20, 30 and $60 \mathrm{~min}$ after the insulin injection, and blood glucose levels were measured using standard test strips (One Touch UltraMini/Johnson \& Johnson, Milpitas, CA, USA) [5]. The ITT is expressed as Kitt (\%/min), i.e., the percent decrease in the plasma glucose concentration per minute [28].

\section{Statistical analysis}

Data are presented as the means \pm SEM. One or two-way ANOVA followed by the Student-Newman-Keuls post hoc test or Student's $t$-test were used for comparisons as appropriate. Differences were considered significant at $P<0.05$.

\section{Experimental protocols}

Rats were used in the following experimental protocols. Body weight was recorded weekly.
Metabolic and cardiovascular responses and gene expression in the NTS in rats fed a HFD

The ITT was performed following an $8 \mathrm{~h}$ fast in rats that had been fed with either SD or HFD for 6 weeks. Two days later, MAP and HR were measured in freely moving rats. On the day following these tests, after $12 \mathrm{~h}$ of fasting, rats were deeply anesthetized, decapitated and blood and brain tissues were collected for analyses of leptin and lipid profiles, and the expression of PIC and RAS genes in the NTS, respectively. Adipose tissue was collected and weighed.

\section{Neuroinflammatory markers in the NTS of rats fed the HFD}

In a separate group of rats, animals were deeply anesthetized after 6 weeks of feeding on the SD or HFD, perfused transcardially and the brainstem was processed for immunohistochemical staining of microglia and astrocytes.

\section{Cardiovascular changes produced by AT1R blockade in the NTS in rats fed with HFD}

After 6 weeks of being fed the SD or HFD, a group of rats underwent surgery to implant cannulas into the iNTS. Rats were maintained on their previous diet (SD or HFD) for the next 7 days for recovery and then polyethylene cannulas were implanted in the femoral artery and vein on the day before MAP and HR recordings in freely moving rats. Baseline MAP and HR were recorded for $\sim 20$ min before starting the tests. Baroreflex function, MAP, HR, and LF of the SBP were evaluated before and $30 \mathrm{~min}$ after the bilateral microinjections of losartan $(10 \mu \mathrm{g} / 100 \mathrm{nl})$ into the NTS.

\section{Cardiovascular changes produced by AT2R overexpression in the NTS in rats fed the HFD}

A group of rats underwent surgery to implant telemetry transmitters into the abdominal aorta. One week later, animals were randomly separated into four groups: two groups were fed the SD and two groups were fed the HFD. After 3 weeks, animals fed the SD and HFD received microinjections of either AAV2-CBA-eGFP or AAV2-CBAAT2R vectors in the NTS, forming the following groups: SD-eGFP, SD-AT2R, HFD-eGFP, and HFD-AT2R. MAP and HR were recorded weekly, starting 1 week before (week 0) and continuing for 6 weeks after commencing the diets. At the end of the experiment, rats were deeply anesthetized, decapitated, and brains were harvested for analyses of PIC and RAS genes in the NTS.

In another group of animals, after 3 weeks of feeding either SD or HFD, rats received microinjections of either AAV2-CBA-eGFP or AAV2-CBA-AT2R into the NTS and 
Table 1 Body weight, adiposity, and plasma measurements in rats fed with standard diet (SD) or high-fat diet (HFD) for 6 weeks

\begin{tabular}{lcc}
\hline & SD & HFD \\
\hline Initial body weight $(\mathrm{g})$ & $305 \pm 2.2(n=9)$ & $301 \pm 3.0(n=10)$ \\
Final body weight $(\mathrm{g})$ & $408 \pm 2.3(n=9)$ & $426 \pm 3.9^{*}(n=10)$ \\
Epididymal adipose tissue (g/100 g body wt.) & $0.73 \pm 0.04(n=9)$ & $1.13 \pm 0.08^{*}(n=10)$ \\
Retroperitoneal adipose tissue (g/100 g body wt.) & $0.61 \pm 0.03(n=9)$ & $0.97 \pm 0.03^{*}(n=10)$ \\
Mesenteric adipose tissue (g/100 g body wt.) & $0.55 \pm 0.07(n=9)$ & $0.96 \pm 0.11^{*}(n=10)$ \\
Total cholesterol (mg/dl) & $60 \pm 2.0(n=6)$ & $80 \pm 2.5^{*}(n=6)$ \\
HDL (mg/dl) & $22 \pm 0.4(n=8)$ & $22 \pm 1.4(n=8)$ \\
TGL (mg/dl) & $39 \pm 2.6(n=8)$ & $56 \pm 5.0^{*}(n=8)$ \\
Blood glucose (mg/dl) & $91 \pm 2.3(n=7)$ & $109 \pm 2.4^{*}(n=7)$ \\
Kitt $(\% / \mathrm{min})$ & $3.7 \pm 0.1(n=7)$ & $2.7 \pm 0.3 *(n=7)$ \\
Leptin (ng/ml) & $1.0 \pm 0.2(n=4)$ & $4.5 \pm 1.2^{*}(n=4)$ \\
\hline Afl
\end{tabular}

All values are presented as means \pm SEM; Student $t$-test. Kitt $(\% / \mathrm{min})$ : percentage of plasma glucose concentration decline per minute. Total cholesterol, HDL, TGL, and leptin levels were measured after $12 \mathrm{~h}$ fasting and glucose levels after $8 \mathrm{~h}$ fasting

$H D L$ high-density lipoprotein, $T G L$ triacylglycerol

*Different from SD; $P<0.05$ were subdivided into the SD-eGFP, SD-AT2R, HFD-eGFP, and HFD-AT2R groups. Three weeks after viral transduction (6 weeks of feeding on the SD or HFD), baroreflex function and cardiovascular variability were evaluated. On the day following these tests and after a $12 \mathrm{~h}$ fast, rats were deeply anesthetized, decapitated, and blood and brain tissues were collected for analyses of leptin and lipid profiles and PIC and RAS genes in the NTS, respectively. Adipose tissue was collected and weighed.

\section{Results}

\section{Changes in body weight, the metabolic profile, MAP, neuroinflammatory markers, and RAS components in the NTS in rats fed the HFD}

Before commencing the diet, body weights of both the SD and HFD groups were similar. Six-weeks after being placed on the diets, the HFD group displayed higher body weight (final body weight), adiposity, and levels of cholesterol, triacylglycerol (TGL), and leptin $(P<0.001)$, whereas high-density lipoprotein (HDL) levels were similar between the groups (Table 1). Blood glucose levels increased and insulin sensitivity decreased after 6 weeks of feeding on the HFD compared to rats fed the SD $(P<0.05)$ (Table 1).

Similar to our previous study [5], the HFD increased MAP (116 \pm 1 vs. SD: $106 \pm 1 \mathrm{mmHg}, P<0.01, n=8)$ and HR $(363 \pm 4$ vs. SD: $338 \pm 7 \mathrm{bpm}, \quad P<0.05 ; \quad n=8)$. Immunoreactivity for GFAP and the number of Iba-1positive cells increased $(P<0.05)$ in the NTS of HFD-fed rats (Fig. 1a, b). Importantly, no differences in immunoreactivity for GFAP (104 \pm 6 vs. SD: $106 \pm 11 ; P=$ $0.895)$ and the number of Iba-1-positive cells (66 \pm 8 vs. SD: $50 \pm 7 ; P=0.204)$ in the AP were observed between the HFD and SD groups. A slight decrease in GFAP immunoreactivity was observed in the hypoglossal nucleus (XII) of the HFD group (HFD $120 \pm 4$ vs. SD: $145 \pm 9 ; P<$ $0.05)$, but the number of Iba-1-positive cells was not significantly different (HFD: $24 \pm 7$ vs. SD: $36 \pm 12 ; P=$ $0.398)$. In addition, in another group of rats, the expression of the mRNAs encoding the PIC TNF- $\alpha$ and IL-6 (Fig. 1c), as well as ACE and AT1R (Supplementary Figure 1), was increased in the NTS of HFD-fed rats, whereas levels of the AT2R and Mas receptor mRNA decreased, without changes in ACE2 expression in the NTS (Supplementary Figure 1), similar to our previous reports [5].

\section{Effects of AT1R blockade in the NTS on cardiovascular parameters in rats fed the HFD}

Bilateral losartan (LOS; $10 \mu \mathrm{g} / 100 \mathrm{nl}$ ) injections into the NTS of HFD-fed rats decreased the MAP $(P<0.05)$ and the low frequency (LF) of SBP $(P<0.01)$, and improved the reflex bradycardia (slope: $-0.98 \pm 0.24$, vs. HFD pre-LOS: $\quad-0.57 \pm 0.25 \mathrm{bpm} / \mathrm{mmHg}, \quad P<0.01)$, without changing the reflex tachycardia (slope: $-2.33 \pm 0.85$ vs. HFD pre-LOS: $\quad-2.43 . \pm 0.37 \mathrm{bpm} / \mathrm{mmHg}, \quad P=0.397$ ) (Fig. 2a-c). The injection of losartan into the NTS produced no changes in SD rats (Fig. $2 \mathrm{a}-\mathrm{c}$ ), and the baseline HR was not different among groups before or after the losartan injections (SD pre-LOS: $313 \pm 9$, SD-post LOS: $313 \pm 10$, HFD pre-LOS: $304 \pm 6$, HFD-post LOS: $299 \pm 6 \mathrm{bpm} ; P=$ $0.164)$. The typical sites of bilateral microinjections into the iNTS are depicted in Fig. 2 d. 

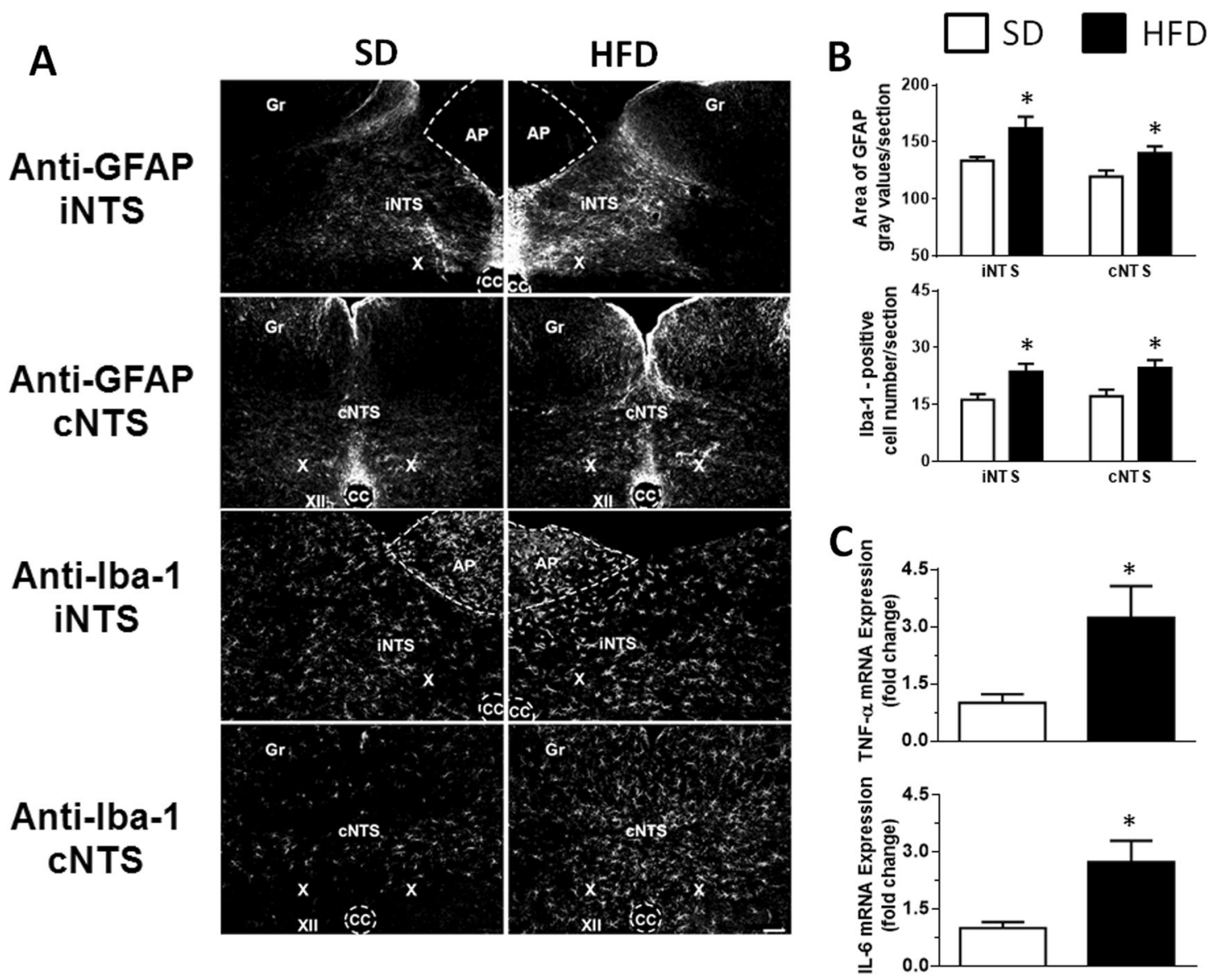

Fig. 1 a GFAP and Iba-1 immunoreactivity in the intermediate NTS (iNTS) and commissural NTS (cNTS) in rats fed either the standard diet (SD; left panels) or high-fat diet (HFD; right panels). Gr gracile nucleus, $\mathrm{X}$ dorsal motor nucleus of the vagus, XII hypoglossal nucleus, CC central canal, AP area postrema. Scale bar $=100 \mu \mathrm{m}$. b Quantification of the area of GFAP staining (gray values/section)

HFD-fed or SD-fed animals in which cannulas were implanted adjacent to the NTS into the hypoglossal nucleus (misplaced injections) showed no changes in MAP after the losartan injections, whereas HR exhibited a slight increase in SD rats (Supplementary Table 1).

\section{Effects of increased expression of AT2R in the NTS on cardiovascular parameters in rats fed the HFD}

All groups exhibited similar baseline levels of MAP (HFDAT2R: $91 \pm 2$ vs. HFD-eGFP: $90 \pm 1$, and SD-AT2R: $91 \pm 2$ vs. SD-eGFP: $90 \pm 1 \mathrm{mmHg}, P>0.05$ ) and HR (HFDAT2R: $304 \pm 4$ vs. HFD-eGFP $310 \pm 3$, and SD-AT2R: $310 \pm 5$ vs. SD-eGFP: $304 \pm 6 \mathrm{bpm}, P>0.05$ ) before the initial implementation of the diet. Three weeks after being fed the HFD, these animals exhibited increases in MAP and HR compared to the SD groups $(P<0.05$; Fig. $3 a)$. AT2R transduction in the NTS of the HFD-fed rats was not effective at altering the increased MAP, but decreased the and number of Iba-1-positive cells in the NTS and $\mathbf{c}$ tumor necrosis factor- $\alpha$ (TNF- $\alpha$ ) and interleukin-6 (IL-6) gene expression in rats fed either the SD or HFD. The results are presented as the means \pm SEM; $n=7$ and $n=6$ animals in the SD and HFD groups shown in $\mathbf{b}$ and c, respectively. Student's $t$-test; *different from the SD group; $P<0.05$

observed tachycardia compared to HFD-eGFP $(P<0.05$; Fig. 3a). The increased expression of AT2R in the NTS of HFD-fed rats attenuated the increase in LF of SBP $(P<$ 0.05; Fig. 3b) and the impairment of the reflex bradycardia (slope: $-1.19 \pm 0.17$, vs. HFD-eGFP: $-0.78 \pm 0.25 \mathrm{bpm} /$ mmHg, $\quad P<0.05$; Fig. 3c). The reflex tachycardia (slope: $-3.18 \pm 0.68$, vs. HFD-eGFP: $-2.86 \pm 0.48$ $\mathrm{bpm} / \mathrm{mmHg}, P=0.139$ ) was not modified by the HFD or by the increased expression of ATR2 alone or in combination with the HFD $(P=0.13$; Fig. 3c).

AT2R transduction into the NTS increased AT2R mRNA levels in SD-fed rats $(1.96 \pm 0.16$, vs. SD-eGFP: $1.0 \pm 0.14$-fold change) and HFD-fed rats $(1.87 \pm 0.11$, vs. HFD-eGFP: $0.99 \pm 0.14$-fold change) $(P<0.05)$. Compared to the changes observed in the NTS of HFD-eGFP rats, HFD-AT2R rats exhibited a reduction in the levels of the ACE mRNA $(P<0.05)$, an increase in the levels of the Mas mRNA $(P<0.05)$ and an increase in the ACE2 mRNA levels $(P<0.05)$, without changing the increased levels of 


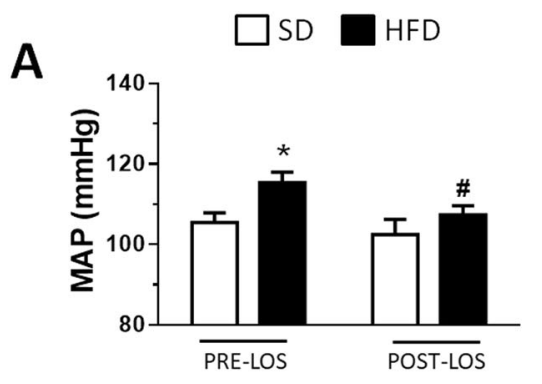

C

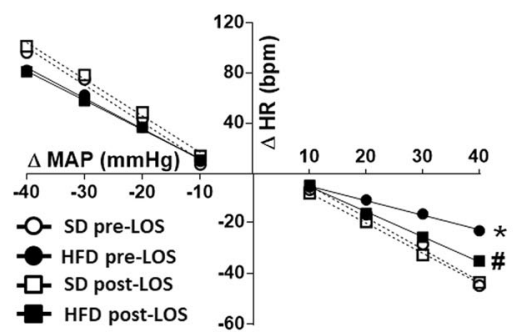

Fig. 2 a Mean arterial pressure (MAP), b low frequency (LF) of the systolic blood pressure (SBP), and c baroreflex function in rats fed either the standard diet (SD) or high-fat diet (HFD) before and after bilateral injections of losartan (LOS; $10 \mu \mathrm{g} / 100 \mathrm{nl}$ ) into the nucleus of the solitary tract (NTS). d Photomicrograph of a coronal section of the brainstem showing the typical location of the intermediate NTS

the AT1R mRNA $(P>0.05)$ (Fig. 4). Moreover, AT2R transduction in the NTS of HFD-fed rats abolished the increased mRNA levels of TNF- $\alpha(P<0.0001)$ and IL-6 $(P<0.05)$ observed in HFD-eGFP rats (Fig. 4). Mas and ACE2 mRNA levels were also increased in SD-AT2R rats (Fig. 4).

After 6 weeks of feeding on the HFD ( 3 weeks of vector injections), the HFD-AT2R and HFD-eGFP rats showed a higher body mass $(P<0.05)$ and higher relative weights of the EPI $(P<0.01)$, RET $(P<0.0001)$, and MES $(P<0.01)$ adipose tissues than their respective control groups (SD groups) (Table 2). Both HFD groups also exhibited higher blood total cholesterol $(P<0.001)$, TGL $(P<0.01)$, and leptin $(P<0.0001)$ levels than their respective control groups (Table 2). No differences in the blood HDL levels were observed between groups $(P=0.40)$. The HFDinduced changes in all variables were comparable within the HFD group (all $P>0.05$ ) (Table 2).

\section{Discussion}

The increase in MAP and in the LF of SBP and impairment of the reflex bradycardia produced by the HFD is consistent with previous studies $[5,8]$. These changes were accompanied by an increase in components of the hypertensive axes and a decrease in components of the protective axes of the RAS in the NTS, as well as neuroinflammation, as

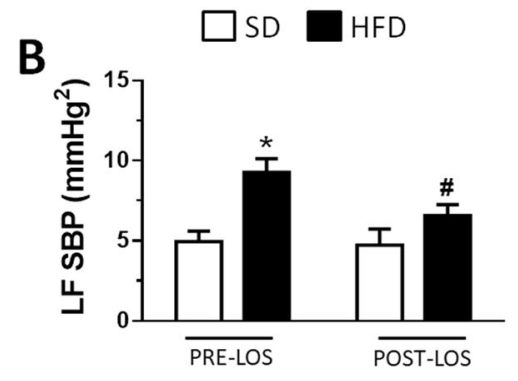

D

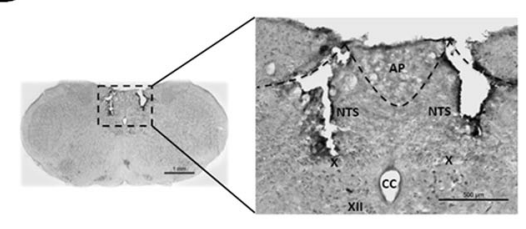

microinjection sites. AP area postrema, CC central canal, $\mathrm{X}$ dorsal motor nucleus of the vagus, XII hypoglossal nucleus. Scale bar $=$ $500 \mu \mathrm{m}$. The results are presented as the means \pm SEM from the SD $(n=5)$ and HFD $(n=6)$ groups. Two-way ANOVA followed by the Student-Newman-Keuls test; *different from the SD group; \#different from the HFD pre-LOS group; $P<0.05$

reported in a previous study from our laboratory [5]. HFDfed animals displayed increased body weight, adiposity, lipid profile, plasma leptin, and blood glucose levels and a decrease in insulin sensitivity, consistent with a previous report [5]. Based on the results of the present study, blockade of AT1R in the NTS acutely abolished the increase in MAP and the LF of SBP (an index of vasomotor sympathetic activity) and the impairment of baroreflex caused by the HFD. The overexpression of AT2R in the NTS of HFD-fed rats reduced the baroreflex impairment, without changing the increase in MAP. In addition, the overexpression of AT2R in the NTS of HFD-fed rats increased the expression of the Mas and ACE2 mRNAs and abolished the HFD-induced increases in the expression of the ACE, TNF- $\alpha$, and IL-6 mRNAs in the same area, without altering the increase in AT1R mRNA expression.

The NTS is more than a synaptic relay for cardiovascular afferents, since it participates in the mechanism mediating the high blood pressure and sympathoexcitation observed in SHRs and in rats with renovascular hypertension [12, 15, 29]. Activation of AT1R in the NTS has been shown to blunt baroreflex function [17] and the overexpression of macrophage migration inhibitory factor, a protein that inhibits the intracellular actions of the AT1R, in the same nuclei improves baroreflex function and lowers blood pressure in SHR and rats with renal hypertension [21, 29]. Obesityinduced hypertension has been treated with different antihypertensive drugs, including AT1R blockers (reviewed in 

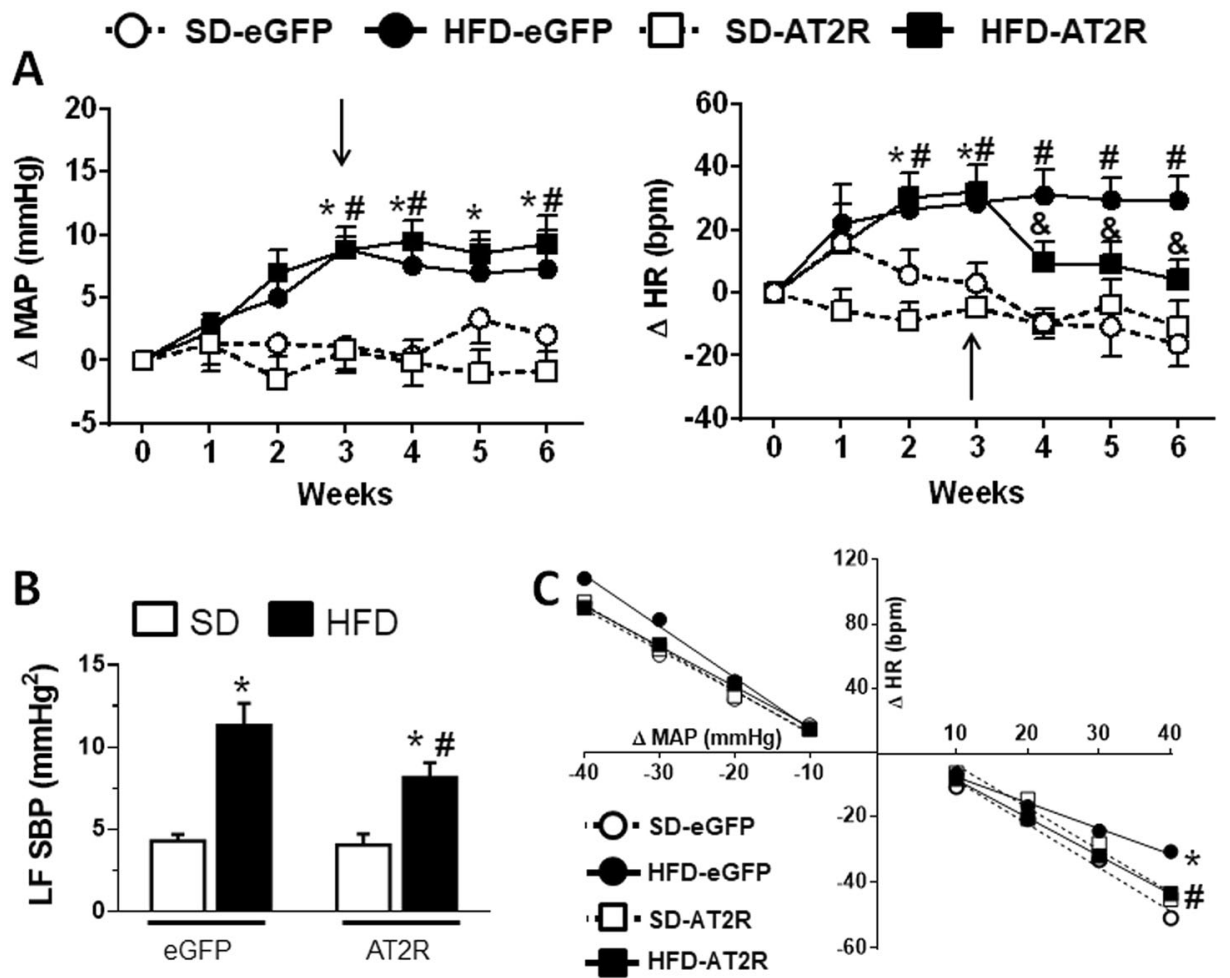

Fig. 3 a Changes in mean arterial pressure (MAP) and heart rate (HR); b low frequency (LF) of the systolic blood pressure (SBP); and c baroreflex function in rats fed either the standard diet (SD) or highfat diet (HFD) that received injections of AAV2-CBA-eGFP or AAV2-CBA-AT2R (arrows in a) into the nucleus of the solitary tract 3 weeks after starting the SD or HFD. The results are presented as the means \pm SEM from the SD-eGFP $(n=6)$, HFD-eGFP $(n=7)$,
SD-AT2 $(n=5)$, and HFD-AT2 $(n=7)$ group. In a, data were analyzed using two-way ANOVA followed by the Student-NewmanKeuls test; *different from week 0; \# different from the SD group; \& different from the HFD-eGFP group; $P<0.05$. In $\mathbf{b}$ and $\mathbf{c}$, data were analyzed using one-way ANOVA followed by the Student-NewmanKeuls test; *different from the SD group; \# different from the HFD-eGFP group; $P<0.05$
Kidambi and Kotchen [30]). In the present study, the blockade of AT1R in the NTS reduced the increased levels of MAP, the elevated LF of SBP and the impairment of the reflex bradycardia observed in HFD-fed rats, suggesting that the activation of AT1R in the NTS is an important mechanism underlying the cardiovascular changes produced in HFD-fed rats. The lack of an effect of losartan injections into areas adjacent to the NTS reinforces the specificity of the NTS in these responses. In addition to the increase in central RAS activity, particularly in the NTS of the HFDfed rats, systemic RAS activity is also increased in these animals [6]. Furthermore, as the blood brain barrier becomes leaky in HFD-fed rats [31], the increased levels of peripheral ANG II in these animals might also reach the NTS and thereby activate AT1R, inducing an increase in MAP and a decrease in baroreflex function.

Baroreflex function has been shown to be a predictor of cardiac mortality [32, 33], and thus impaired baroreflex function should also be considered for neurogenic hypertension treatment. In this sense, AT2R overexpression improved the baroreflex function, similar to the observations in our previous study using the renovascular [12] and
SHR [13] hypertension models. Furthermore, transduction of AT2R into the NTS increased the levels of Mas receptors and ACE2 expressed at this site in HFD-fed rats. Studies have demonstrated that ANG (1-7) in the NTS improves baroreflex function and reduces arterial pressure [34], whereas blockade of ACE2 in the NTS reduces baroreflex function [35]. Since the AT1R and AT2R expressed in the NTS are mostly localized in different neurons, with only a $7 \%$ overlap, according to a previous study [19], the data might suggest that AT1R inhibition and AT2R activation function through different mechanisms/circuits to dampen the cardiovascular system in hypertension. An open question is if the intra-NTS application of an AT2R agonist would improve the baroreflex function in HFD-fed animals. In rats with heart failure displaying a decreased level of AT2R in the rostral ventrolateral medulla (RVLM), the injection of an AT2R agonist in the RVLM induces a smaller decrease in MAP than in control rats [36]. As HFDfed rats exhibit decreased expression of the AT2R mRNA in the NTS, an acute injection of an AT2R agonist in the NTS may not improve the baroreflex, but future studies are needed to address this question. 
Fig. 4 a Angiotensin II type 1 receptor (AT1R), Mas receptor, angiotensin-converting enzyme (ACE), and angiotensinconverting enzyme 2 (ACE2) mRNA levels, and $\mathbf{b}$ tumor necrosis factor- $\alpha(\mathrm{TNF}-\alpha)$ and interleukin-6 (IL-6) mRNA levels in rats fed the standard diet (SD) or high-fat diet (HFD) that received injections of AAV2-CBA-eGFP or AAV2CBA-AT2R into the NTS. The results are presented as the means \pm SEM; $n=7$ animals in all groups. One-way ANOVA followed by the

Student-Newman-Keuls test; *different from the SD group; \# different from the HFD-eGFP group; \& different from the SDeGFP group; $P<0.05$
A
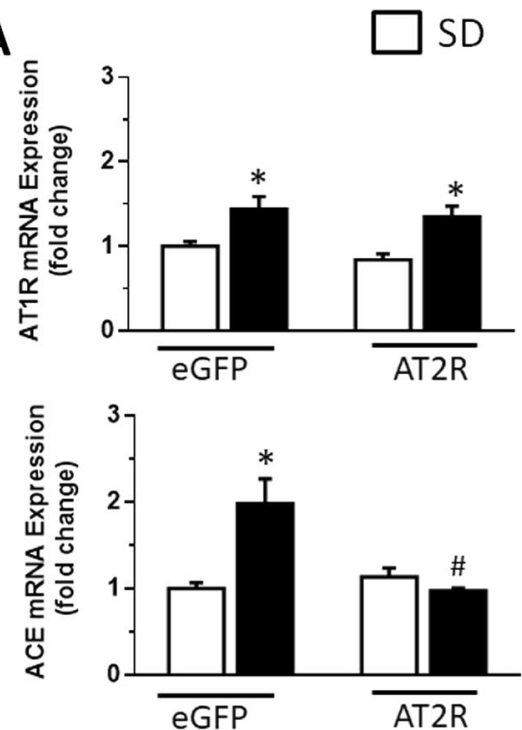

B

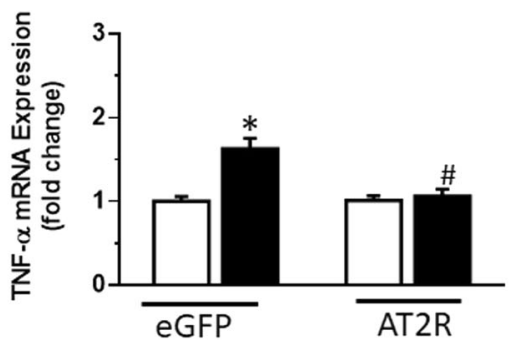

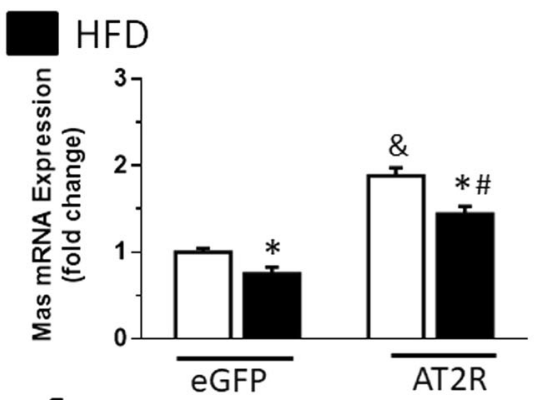
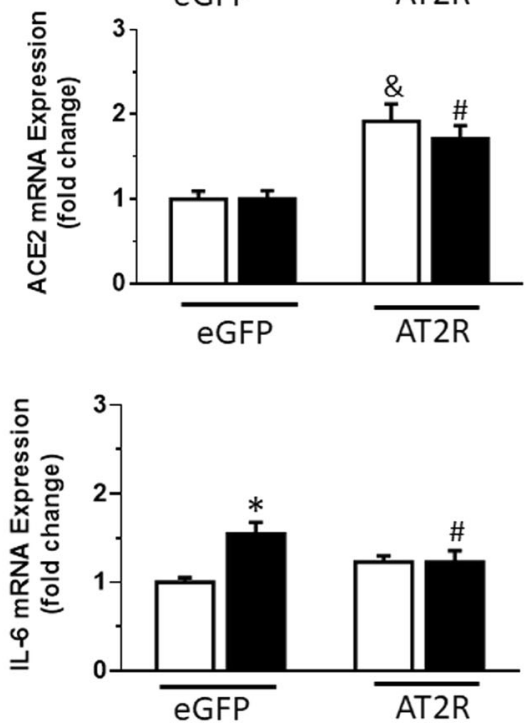

As shown in our previous study, AT2R overexpression in the NTS reduces hypertension in rats with renovascular hypertension [12]. In the present study, a slight decrease in the LF of SBP was observed in the HFD-AT2R group, but the effect was probably not sufficient to restore MAP. In addition, the LF of SPB is an indirect measurement of the SNA, and therefore has its own limitations. Thus, this slight decrease may not accurately reflect a reduction in SNA that would impact the peripheral resistance. In HFD-AT2R rats, we observed a decrease in baseline HR ( 20 bpm). The baroreceptor reflex pathways provide a major excitatory drive to the cardiac vagal activity [37], thus the decrease in baseline HR might have been secondary to the improvement in the baroreflex. Interestingly, in the HFD-AT2R group, no change in the elevated levels of AT1R in the NTS was observed. Further studies are required to determine whether another mechanism activated by AT1R in the NTS of HFD is also involved under these conditions to maintain the higher levels of MAP, such as an increase in heart contractility or a humoral factor.

In the present study, we observed an increased number of microglia and astrocytes in the NTS of HFD-fed rats that was associated with higher expression of the TNF- $\alpha$ and IL6 mRNAs, as previously reported [5]. Since cross-talk between the RAS and the immune system in the brain has been suggested, including during obesity [3, 18], an interplay between the RAS and PICs may also exist in the NTS and account for the change in baroreflex function in HFDfed rats. In fact, IL-6 microinjections into the NTS attenuate baroreflex function [38], and the reduction in the IL-6 mRNA levels observed after overexpressing AT2R in the NTS might also account for the improvement in the baroreflex response observed in the HDF-fed rats. Conversely, TNF- $\alpha$ injections in the NTS fail to induce any change in MAP [39], and thus the role of the increased levels of TNF$\alpha$ in the NTS remains to be determined. Importantly, the HFD induces different degrees of neuroinflammation in different areas of the brain [40]. In the present study, we also evaluated the expression of microglial and astrocyte markers in the XII and AP, which are adjacent to the NTS. No significant changes in either area were observed, indicating that, at least for the diet used in the present study, the inflammation occurring in the dorsal brainstem seems to be more specific to the NTS.

Obesity is a multifactorial disease, and the alterations in lipid profile, insulin resistance, and hyperleptinemia observed in rats fed the HFD may contribute to the increased MAP (reviewed in ref. [41]). For instance, insulin and leptin acting in the forebrain increase SNA [7, 42, 43] and an interaction between ANG II, leptin, ANG II and 
Table 2 Body weight, adiposity, and plasma measurements in rats fed with standard chow diet (SD) or high-fat diet (HFD) for 6 weeks along with transduction in the nucleus of the solitary tract of GFP (SD-eGFP and HFD-eGFP) or AT2R (SD-AT2R and HFD-AT2R)

\begin{tabular}{lcccc}
\hline & SD-eGFP & HFD-eGFP & SD-AT2R & HFD-AT2R \\
\hline Initial body mass $(\mathrm{g})$ & $309 \pm 1(n=6)$ & $307 \pm 1(n=7)$ & $309 \pm 1(n=5)$ & $306 \pm 1(n=7)$ \\
Final body mass $(\mathrm{g})$ & $387 \pm 13(n=6)$ & $471 \pm 9 *(n=7)$ & $393 \pm 12(n=5)$ & $440 \pm 13^{\mathrm{a}}(n=7)$ \\
Epididymal adipose tissue (g/100 g body mass) & $0.35 \pm 0.03(n=6)$ & $0.91 \pm 0.07 *(n=7)$ & $0.45 \pm 0.06(n=5)$ & $0.80 \pm 0.06^{*}(n=7)$ \\
Retroperitoneal adipose tissue (g/100 g body mass) & $0.43 \pm 0.03(n=6)$ & $1.18 \pm 0.08^{*}(n=7)$ & $0.47 \pm 0.05(n=5)$ & $1.16 \pm 0.08^{*}(n=7)$ \\
Mesenteric adipose tissue (g/100 g body mass) & $0.44 \pm 0.05(n=6)$ & $0.83 \pm 0.06^{*}(n=7)$ & $0.39 \pm 0.03(n=5)$ & $0.70 \pm 0.03 *(n=7)$ \\
Total cholesterol (mg/dl) & $64 \pm 5.7(n=6)$ & $93 \pm 2.5^{*}(n=7)$ & $60 \pm 2.4(n=5)$ & $86 \pm 3.23^{*}(n=7)$ \\
HDL (mg/dl) & $38 \pm 2.3(n=6)$ & $38 \pm 3.3(n=7)$ & $34 \pm 2.2(n=5)$ & $40 \pm 1.5(n=7)$ \\
TGL (mg/dl) & $54 \pm 4.4(n=6)$ & $79 \pm 2.9 *(n=7)$ & $51 \pm 3.9(n=5)$ & $84 \pm 6.1 *(n=7)$ \\
Leptin (ng/ml) & $0.48 \pm 0.12(n=4)$ & $1.89 \pm 0.20^{*}(n=6)$ & $0.39 \pm 0.05(n=4)$ & $2.55 \pm 0.28^{*}(n=5)$ \\
\hline
\end{tabular}

All values are presented as means \pm SEM. One-way ANOVA followed by the Student-Newman-Keuls test. Total cholesterol, HDL, TGL, and leptin levels were measured after $12 \mathrm{~h}$ fasting

$H D L$ high-density lipoprotein, $T G L$ triacylglycerol

*Different from SD; $P<0.05$

insulin has been reported in the forebrain [7, 44, 45]. The present results revealed a pronounced involvement of AT1R in the NTS in the cardiovascular changes observed after 6 weeks of feeding on the HFD. If an interaction exists between angiotensinergic mechanisms and leptin/insulin within the NTS to control these cardiovascular changes, as observed in the forebrain, it remains to be determined.

In conclusion, AT1R activation in the NTS is an important mechanism underlying the increase in MAP, whereas AT1R and AT2R may affect baroreflex function in opposite directions in HFD-fed rats. Chronic inflammation and changes in the RAS in the NTS may also account for the cardiovascular responses observed in HFD-fed rats. We did not clearly determine whether the recovery of baroreflex function in HFD-fed rats overexpressing AT2R was a direct effect of the increase of AT2R in the NTS or was mediated by indirect effects via reducing neuroinflammation and/or increasing the protective axis of the RAS in the NTS.

Acknowledgements The authors thank Dr. Rodrigo Tomeo and Dr. Regina C. Vendramini for providing expert technical assistance, Silvana A.D. Malavolta and Carla D. Alencar for providing secretarial assistance and Ana V. Oliveira for providing animal care. This study was supported by Fundação de Amparo à Pesquisa do Estado de São Paulo [2013/13118-0; 2015/234677]; Conselho Nacional de Desenvolvimento Científico e Tecnológico [304873/2014-4; 425586/2016-2; 308099/2017-6]; Coordenação de Aperfeiçoamento de Pessoal de Nível Superior [33001014037P4 and Finance Code 001]; and NIH grant HL-07803.

\section{Compliance with ethical standards}

Conflict of interest The authors declare that they have no conflict of interest.

Publisher's note: Springer Nature remains neutral with regard to jurisdictional claims in published maps and institutional affiliations.

\section{References}

1. de Kloet AD, Krause EG, Shi PD, Zubcevic J, Raizada MK, Sumners C. Neuroimmune communication in hypertension and obesity: a new therapeutic angle? Pharmacol Ther. 2013;138: 428-40.

2. Leggio M, Lombardi M, Caldarone E, Severi P, D'Emidio S, Armeni M, et al. The relationship between obesity and hypertension: an updated comprehensive overview on vicious twins. Hypertens Res. 2017;40:947.

3. de Kloet AD, Pioquinto DJ, Nguyen D, Wang L, Smith JA, Hiller $\mathrm{H}$, et al. Obesity induces neuroinflammation mediated by altered expression of the renin-angiotensin system in mouse forebrain nuclei. Physiol Behav. 2014;136:31-38.

4. Speretta GF, Rosante MC, Duarte FO, Leite RD, Lino AD, Andre RA, et al. The effects of exercise modalities on adiposity in obese rats. Clinics. 2012;67:1469-77.

5. Speretta GF, Silva AA, Vendramini RC, Zanesco A, Delbin MA, Menani JV, et al. Resistance training prevents the cardiovascular changes caused by high-fat diet. Life Sci. 2016;146:154-62.

6. Boustany CM, Bharadwaj K, Daugherty A, Brown DR, Randall DC, Cassis LA. Activation of the systemic and adipose reninangiotensin system in rats with diet-induced obesity and hypertension. Am J Physiol Regul Integr Comp Physiol. 2004;287: R943-R949.

7. Hilzendeger AM, Morgan DA, Brooks L, Dellsperger D, Liu X, Grobe JL, et al. A brain leptin-renin angiotensin system interaction in the regulation of sympathetic nerve activity. Am J Physiol Heart Circ Physiol. 2012;303:H197-H206.

8. Fardin NM, Oyama LM, Campos RR. Changes in baroreflex control of renal sympathetic nerve activity in high-fat-fed rats as a predictor of hypertension. Obesity. 2012;20:1591-7.

9. Grassi G, Seravalle G, Dell'Oro R, Turri C, Bolla GB, Mancia G. Adrenergic and reflex abnormalities in obesity-related hypertension. Hypertension. 2000;36:538-42.

10. Guimaraes PS, Huber DA, Campagnole-Santos MJ, Schreihofer AM. Development of attenuated baroreflexes in obese Zucker rats coincides with impaired activation of nucleus tractus solitarius. Am J Physiol Regul Integr Comp Physiol. 2014;306:R681-R692.

11. Talman WT, Perrone MH, Reis DJ. Evidence for L-glutamate as the neurotransmitter of baroreceptor afferent nerve fibers. Science. 1980;209:813-5. 
12. Blanch GT, Freiria-Oliveira AH, Speretta GF, Carrera EJ, Li H, Speth RC, et al. Increased expression of angiotensin II Type 2 receptors in the solitary-vagal complex blunts renovascular hypertension. Hypertension. 2014;64:777-83.

13. Ruchaya PJ, Speretta GF, Blanch GT, Li H, Sumners C, Menani $\mathrm{JV}$, et al. Overexpression of AT2R in the solitary-vagal complex improves baroreflex in the spontaneously hypertensive rat. Neuropeptides. 2016;60:29-36.

14. Ciriello J, Hochstenbach SL, Roder S. Central projections of baroreceptor and chemoreceptor afferents fibers in the rat. In: Barraco IRA, editor. Nucleus of the solitary tract. Boca Raton, FL: CRC Press; 1994. p. 35-50.

15. Colombari E, Sato MA, Cravo SL, Bergamaschi CT, Campos RR $\mathrm{Jr}$, Lopes OU. Role of medulla oblongata in hypertension. Hypertension. 2001;38:549-54.

16. Allen AM, McKinley MJ, Oldfield BJ, Dampney RA, Mendelsohn FA. Angiotensin II receptor binding and the baroreflex pathway. Clin Exp Hypertens A. 1988;10(Suppl. 1):63-78.

17. Paton JF, Kasparov S. Differential effects of angiotensin II on cardiorespiratory reflexes mediated by nucleus tractus solitarii-a microinjection study in the rat. J Physiol. 1999;521:213-25.

18. Xue B, Thunhorst RL, Yu Y, Guo F, Beltz TG, Felder RB, et al. Central renin-angiotensin system activation and inflammation induced by high-fat diet sensitize angiotensin II-elicited hypertension. Hypertension. 2016;67:163-70.

19. de Kloet AD, Wang L, Ludin JA, Smith JA, Pioquinto DJ, Hiller $\mathrm{H}$, et al. Reporter mouse strain provides a novel look at angiotensin type-2 receptor distribution in the central nervous system. Brain Struct Funct. 2016;221:891-912.

20. Lenkei Z, Palkovits M, Corvol P, Llorens-Cortes C. Expression of angiotensin type-1 (AT1) and type-2 (AT2) receptor mRNAs in the adult rat brain: a functional neuroanatomical review. Front Neuroendocrinol. 1997;18:383-439.

21. Barbosa RM, Speretta GF, Dias DPM, Ruchaya PJ, Li H, Menani $\mathrm{JV}$, et al. Increased expression of macrophage migration inhibitory factor in the nucleus of the solitary tract attenuates renovascular hypertension in rats. Am J Hypertens. 2017;30:435-43.

22. Cardoso LM, Colombari DS, Menani JV, Toney GM, Chianca DA Jr., et al. Cardiovascular responses to hydrogen peroxide into the nucleus tractus solitarius. Am J Physiol Regul Integr Comp Physiol. 2009;297:R462-R469.

23. Vieira AA, Colombari E, De Luca LA Jr, Colombari DSA, De Paula PM, Menani JV. Importance of angiotensinergic mechanisms for the pressor response to l-glutamate into the rostral ventrolateral medulla. Brain Res. 2010;1322:72-80.

24. Zhang Y, Gao Y, Speth RC, Jiang N, Mao Y, Sumners C, et al. Adenoviral and adeno-associated viral vectors-mediated neuronal gene transfer to cardiovascular control regions of the rat brain. Int J Med Sci. 2013;10:607-16.

25. Livak KJ, Schmittgen TD. Analysis of relative gene expression data using real-time quantitative PCR and the 2(-Delta Delta C(T)) Method. Methods. 2001;25:402-8.

26. Blanch GT, Freiria-Oliveira AH, Murphy D, Paulin RF, AntunesRodrigues J, Colombari E, et al. Inhibitory mechanism of the nucleus of the solitary tract involved in the control of cardiovascular, dipsogenic, hormonal, and renal responses to hyperosmolality. Am J Physiol Regul Integr Comp Physiol. 2013;304:R531-R542.

27. Bonora E, Moghetti P, Zancanaro C, Cigolini M, Querena M, Cacciatori V, et al. Estimates of in vivo insulin action in man: comparison of insulin tolerance tests with euglycemic and hyperglycemic glucose clamp studies. J Clin Endocrinol Metab. 1989;68:374-8.

28. Delbin MA, Davel AP, Couto GK, de Araujo GG, Rossoni LV, Antunes $\mathrm{E}$, et al. Interaction between advanced glycation end products formation and vascular responses in femoral and coronary arteries from exercised diabetic rats. PLoS One. 2012;7: e53318.

29. Freiria-Oliveira AH, Blanch GT, Li H, Colombari E, Colombari DSA, Sumners C. Macrophage migration inhibitory factor in the nucleus of solitary tract decreases blood pressure in SHRs. Cardiovasc Res. 2013;97:153-60.

30. Kidambi S, Kotchen TA. Treatment of hypertension in obese patients. Am J Cardiovasc Drugs. 2013;13:163-75.

31. Mauro C, De Rosa V, Marelli-Berg F, Solito E. Metabolic syndrome and the immunological affair with the blood-brain barrier. Front Immunol. 2014;5:677.

32. La Rovere MT, Bigger JT Jr., Marcus FI, Mortara A, Schwartz PJ. Baroreflex sensitivity and heart-rate variability in prediction of total cardiac mortality after myocardial infarction. ATRAMI (Autonomic Tone and Reflexes After Myocardial Infarction) Investigators. Lancet. 1998;351:478-84.

33. La Rovere MT, Pinna GD, Maestri R, Sleight P. Clinical value of baroreflex sensitivity. Neth Heart J. 2013;21:61-63.

34. Chaves GZ, Caligiorne SM, Santos RA, Khosla MC, Campagnole-Santos MJ. Modulation of the baroreflex control of heart rate by angiotensin-(1-7) at the nucleus tractus solitarii of normotensive and spontaneously hypertensive rats. J Hypertens. 2000;18:1841-8.

35. Diz DI, Garcia-Espinosa MA, Gegick S, Tommasi EN, Ferrario $\mathrm{CM}$, Ann Tallant E, et al. Injections of angiotensin-converting enzyme 2 inhibitor MLN4760 into nucleus tractus solitarii reduce baroreceptor reflex sensitivity for heart rate control in rats. Exp Physiol. 2008;93:694-700.

36. Gao L, Wang WZ, Wang W, Zucker IH. Imbalance of angiotensin type 1 receptor and angiotensin II type 2 receptor in the rostral ventrolateral medulla: potential mechanism for sympathetic overactivity in heart failure. Hypertension. 2008;52:708-14.

37. Kirchheim HR. Systemic arterial baroreceptor reflexes. Physiol Rev. 1976;56:100-77.

38. Takagishi M, Waki H, Bhuiyan ME, Gouraud SS, Kohsaka A, Cui $\mathrm{H}$, et al. IL-6 microinjected in the nucleus tractus solitarii attenuates cardiac baroreceptor reflex function in rats. Am J Physiol Regul Integr Comp Physiol. 2010;298:R183-R190.

39. Mollace V, Muscoli C, Palma E, Iannone M, Granato T, Nistico $\mathrm{R}$, et al. Central cardiovascular responses induced by interleukin $1 \beta$ and tumor necrosis factor $\alpha$ infused into nucleus tractus solitarii, nucleus parabrachialis medialis and third cerebral ventricle of normotensive rats. Neurosci Lett. 2001;314:53-56.

40. Guillemot-Legris O, Muccioli GG. Obesity-Induced Neuroinflammation: Beyond the Hypothalamus. Trends Neurosci. 2017;40:237-53.

41. Despres JP, Lemieux I. Abdominal obesity and metabolic syndrome. Nature. 2006;444:881-7.

42. Muntzel MS, Morgan DA, Mark AL, Johnson AK. Intracerebroventricular insulin produces nonuniform regional increases in sympathetic nerve activity. Am J Physiol. 1994;267(5 Pt 2): R1350-1355.

43. Mark AL, Agassandian K, Morgan DA, Liu X, Cassell MD, Rahmouni K. Leptin signaling in the nucleus tractus solitarii increases sympathetic nerve activity to the kidney. Hypertension. 2009;53:375-80.

44. Mayer MA, Hocht C, Giani JF, Munoz MC, Carranza A, Taira $\mathrm{CA}$, et al. Central insulin-angiotensin II interaction in blood pressure regulation in fructose overloaded rats. Regul Pept. 2013;185:37-43.

45. Xue B, Yu Y, Zhang Z, Guo F, Beltz TG, Thunhorst RL, et al. Leptin Mediates High-Fat Diet Sensitization of Angiotensin IIElicited Hypertension by Upregulating the Brain ReninAngiotensin System and Inflammation. Hypertension. 2016; 67:970-6. 